\title{
Fibrin(ogen) and diabetes mellitus: don't forget fibrinolysis
}

\author{
M . M orale, F. D e N egri, F. C armassi \\ 2nd Medical Clinic, University of Pisa, Pisa, Italy
}

In recent years several studies have addressed the possible involvement of fibrinogen as an important risk factor for the development of cardiovascular disease. In the present issue of Diabetologia, Dr. Ceriello reviews recent studies, enriched with personal data, on the role of fibrinogen as a risk factor for vascular disease in diabetic patients, who are known to undergo early atherosclerotic complications. Dr. Ceriello poses many questions and, whenever possible, offers a convincing answer. Although many hypotheses need further demonstration, with the data to hand we have no arguments against it; instead, we would like to strengthen his approach by placing emphasis on particular aspects not extensively covered by Dr. Ceriello.

\section{A therosclerosis and inflammation}

The non-answered question about the role of fibrinogen in atherosclerosis is whether increased fibrinogen per se is responsible for cardiovascular risk or it is merely a marker of the underlying vascular disease. Indeed fibrinogen, which is an acute phase reactant, could be only a marker of the inflammatory process which is likely to play a role in atherosclerosis. Many observations can support this hypothesis.

In the inflammatory state fibrinogen increases with peripheral leukocyte count [1]. Leukocytes increase fibrinogen synthesis by secreting interleukin-6 under stimulus from fibrinogen degradation products [2]. The pathogenetic role of inflammation in the development of atherosclerosis is supported by recent studies showing that interleukin-1 and tumour

Corresponding author: Dr. F. Carmassi, 2nd Medical Clinic, University of Pisa, I-56126 Pisa, Italy

A bbreviations: PAI-1, Plasminogen activator inhibitor-1. necrosis factor are able to reverse the functional non-thrombogenic properties of normal endothelium towards prothrombotic changes, enhancing the generation of thrombin at the endothelial surface [3-6]. Polymorphonuclear leukocytes can activate platelets [6], which stimulate the adherence of monocytes to surfaces [5] and the endothelial expression of leukocyte adhesion molecules [7]. Therefore, it appears that during inflammation the endothelium displays properties similar to those of the platelet surface, becoming a thrombogenic environment. In atherogenesis the insudation of lipids in the arterial wall could induce a local inflammatory reaction, with production of proteolytic enzymes, cytokines and growth factors (platelet derived growth factor, etc.), which in turn could amplify the inflammatory process and induce the proliferative reaction of fibroblasts and smooth muscle cells [8]. The release of elastase by activated granulocytes in atherosclerotic plaques has been recently taken as a marker of coronary artery disease, since elastase in plasma appeared to be higher in patients than in control subjects and in patients with complex atheromatous plaques than in those with simple plaques [9].

Inflammation is associated with both hypercoagulability (increased fibrinogen, factor VIII, platelets) [1-7] and hypofibrinolysis (increased expression of plasminogen activator inhibitor-1, PAI-1) [10]. Plasma levels of fibrinogen were found to be associated with the severity of atheromatous involvement of the arterial walls [11]. There is constant deposition and lysis of fibrin within the atherosclerotic arterial wall, and in animal models the amount of fibrin deposited in the arterial walls appeared to be dependent on fibrinogen concentrations [12]. The inverse relationship between fibrinogen concentrations and clot deformability [13] and the increased platelet aggregability in the presence of high levels of fibrinogen [14] appear to contradict the previous opinion that 
fibrinogen levels exceeding those needed for normal hemostasis could not increase thrombogenesis.

To strengthen the importance of clotting in inflammation, we wish to report recent data from Yamamoto et al. [15], showing that clotting of normal human plasma caused the generation of a monocyte chemotactic factor with an apparent molecular mass of $45 \mathrm{kDa}$. The generation of the chemotactic activity was not observed in clotting of plasma deficient in blood coagulation Factor XIII (fibrin-stabilizing factor), but it normally appeared when the deficient plasma was reconstituted with Factor XIII or a tissue transglutaminase [15]. Upon activation by thrombin, Factor XIII catalyses $\alpha$ - and $\gamma$-chain polymerization of fibrin monomers, conferring fibrin mechanical strength and elasticity; besides, it appears to play a crucial role in fibrinolysis by catalysing the crosslink of $\alpha 2$-plasmin inhibitor, the primary inhibitor of plasmin and Factor XIIIa best substrate, into $\alpha$-chain of fibrin, providing early protection of fibrin from premature lysis by plasmin [16-18]. Given the above evidence, Yamamoto et al. [15] likely discovered a novel mechanism which links the transglutaminase of the clotting system with inflammation.

\section{Role of fibrinolysis in atherosclerosis}

An important issue being debated is whether the multitude of epidemiological and clinical studies provide insight into the mechanisms of atherosclerosis or merely reflect the consequences of existing artery disease. In other words, is the fibrinolytic derangement a consequence of inflammation and endothelial dysfunction or a constitutive abnormality predisposing the atherosclerosis and thrombosis? The unifying hypothesis could be that, after the endothelial dysfunction is triggered by inflammation, increased plasma [19] or plaque [20] PAI-1 levels might contribute to aggravate the underlying vessel injury by further promotion of thrombosis and matrix deposition. With current data it is likely that plasminogen/plasmin system is involved in the development and progression of atherosclerosis [13, 19]. Epidemiological studies showed that impaired fibrinolysis and increased level of PAI-1 correlated with re-infarction and degree of coronary heart disease [19]. Further support for such a hypothesis comes from recent studies with transgenic mice over- or under-expressing components of the fibrinolytic system. Mice with 'fibrinolytic knock-out' for the suppression of t-PA or u-PA gene function were found to be more susceptible to the development of thrombosis induced by endotoxin-mediated endothelial damage and developed accelerated neointima formation after vascular trauma [21]. Cellular, fibrin and platelet-rich venous occlusion was also observed in transgenic mice over-expressing PAI-1 [21].

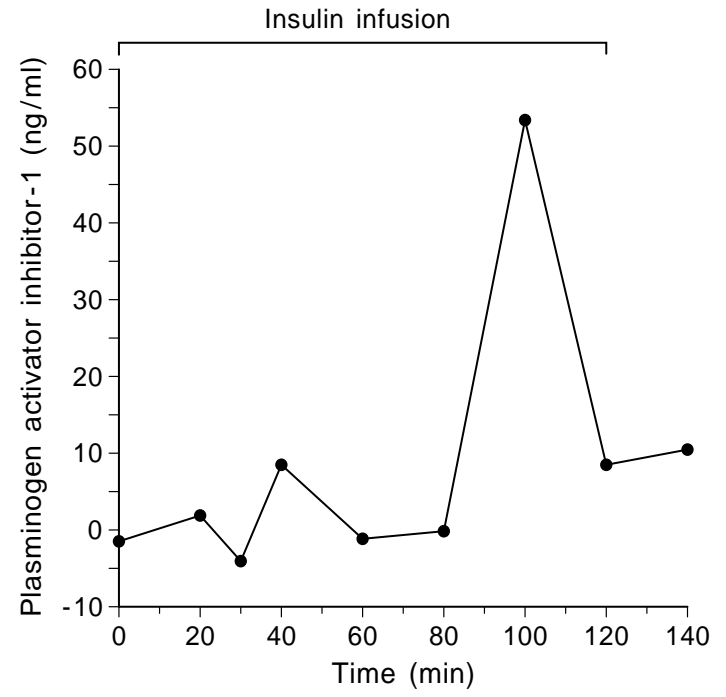

Fig. 1. Changes in veno-arterial concentration of PAI-1 antigen during infusion of insulin $(100 \mu \mathrm{IU} / \mathrm{ml}$ of plasma)

Finally, to complete such a complex scenario, the last glance should be at the interesting but unresolved issue of atherosclerotic activity of lipoprotein(a) $(\mathrm{Lp}(\mathrm{a}))$. The selective affinity between interacting molecules as a means of regulating a specific reaction has been recognized: i.e. plasminogen binding to fibrin [22], fibrinogen binding to plasminogen [23]. Given the striking similarities between the amino acid sequence of apoprotein(a) and the structure of plasminogen [24], the competitive inhibition of the binding of plasminogen to fibrinogen by $\mathrm{Lp}$ (a) might reduce fibrinolysis both in blood and on cell surfaces.

\section{What is the role of fibrinolysis in diabetes?}

Given the above evidence, the same conclusions should hold true for early atherosclerosis in diabetes, where high levels of PAI-1 have been shown in patients with IDDM at risk for atherothrombotic complications [25]. A number of studies have shown the correlation between high plasma level of PAI-1 and hyperinsulinism [26], a feature of NIDDM diabetes, obesity and possibly of IDDM patients on insulin treatment. In euglycaemic clamp studies, insulin infusion appeared to increase the plasma level of PAI-1 [27]. In order to rule out the contribution of hepatocytes to the release of PAI-1 in the systemic circulation and to assess the endothelial response to the infusion of insulin, we used a human model of 'functionally isolated' vascular district where the brachial artery and vein were cannulated, so that veno-arterial difference in PAI-1 concentration could reasonably represent the net production of endothelial PAI-1. A twofold expression of PAI-1 was observed after a one and a half hour infusion of insulin to a final concentration of $100 \mu \mathrm{IU} / \mathrm{ml}$ (Fig. 1). If confirmed, these 
results suggest that increased endothelial expression of PAI-1 induced by insulin could cause a shift in the balance between fibrinolysis and coagulation toward the latter, contributing to the accumulation of fibrin within the arterial walls and therefore to the progression of atherosclerotic disease. The finding of high plasma levels of thrombin-antithrombin III complexes in IDDM patients [25] further strengthens the notion that increased thrombin generation for oxidative stress [28] or hyperglycaemia might cause excess intravascular fibrin production, either directly or by inducing PAI-1 overexpression by endothelial cells.

\section{Conclusions}

Epidemiological, clinical and experimental observations suggest that both enhanced fibrin generation and poor fibrinolysis could play a role in atherosclerosis and its complications. Patients with diabetes reportedly show a complex pattern consistent with the proneness to generate fibrin poorly degradable by plasmin, likely for the impairment of fibrinolytic potential. Fibrinogen, according to Dr. Ceriello, appears a strong predictor of cardiovascular disease in diabetes. Thus, the answer could be: let us lower fibrinogen! Unfortunately, to what extent and at what stage hyperfibrinogenaemia, clotting and lysis of fibrin(ogen) might affect the atherosclerotic lesions should be clarified further and we do not feel comfortable supporting such an idea, especially because at present we lack a drug which can act selectively to lower fibrinogen. Besides, the possibility that hyperfibrinogenaemia is an epiphenomenon should not be disregarded. Given the extraordinary opportunity disclosed by genetic engineering, efforts could be better addressed to challenge single gene deficiency (tPA deficiency, plasminogen deficiency) with different pathological situations such as inflammation, hyperglycaemia and atherosclerosis. Before any pharmacological approach, high priority should be given to define the role of fibrinolysis in atherosclerosis, since the modulation of the fibrinolytic system could be the right approach to prevention.

\section{References}

1. Folsom AR, Wu KK, Davis CE, Conlan MG, Sorlie PD, Szklo M (1991) Population correlates of plasma fibrinogen and factor VII, putative cardiovascular risk factors. Atherosclerosis 91: 191-205

2. Ritchie DG, Levy BA, Adama MA, Fuller GM (1982) Regulation of fibrinogen synthesis by plasmin-derived fragments of fibrinogen and fibrin: an indirect feedback pathway. Proc Natl Acad Sci USA 79: $1530-1534$

3. Stern D, Bank I, Nawroth P et al. (1985) Self-regulation of procoagulant events on the endothelial cell surface. J Exp Med 162: 12231228

4. Nawroth P, Stern D (1986) Modulation of endothelial cell hemostatic properties by tumor necrosis factor. J Exp Med 164: 740-749
5. Burns GF, Cosgrove L, Triglia Tet al. (1986) The IIb-IIIa glycoprotein complex that mediates platelet aggregation is directly implicated in leukocyte adhesion. Cell 45: 269-280

6. Cerletti C, Evangelista V, Molino M, De Gaetano G (1995) Platelet activation by polymorphonuclear leukocytes: role of cathepsin $\mathrm{G}$ and P-selectin. Thromb Haemost 74: 218-223

7. Hawrylowicz CM, Howells GL, Feldmann M (1991) Platelet-derived interleukin-1 induces human endothelial adhesion molecule expression and cytokine expression. J Exp Med 174: 785-790

8. Ross R (1995) Atherogenesis. In: Gallin JI, Goldstein IM, Snyderman R (eds) Inflammation: basic principles and clinical correlates. 2nd edition, Raven Press, New York, pp 1051-1059

9. Amaro A, Gude F, Gonzalez-Juanatey R, Iglesias C, FernandezVazquez F, Garcia-Acuña J, Gil M (1995) Plasma leukocyte elastase concentration in angiographically diagnosed coronary artery disease. Europ Heart J 16: 615-622

10. Juhan-Vague I, Moerman B, De Coick F, Aillaud MF, Collen D (1984) Plasma levels of a specific inhibitor of tissue-type plasminogen activator (and urokinase) in normal and pathological conditions. Thromb Res 33: 523-530

11. Broadhurst P, Kelleher C, Hughes L, Imeson JD, Raftery EB (1990) Fibrinogen, factor VII clotting activity and coronary artery disease severity. Atherosclerosis 85: 169-173

12. Chooi CC, Gallus AS (1989) Acute phase reaction, fibrinogen level and thrombus size. Thromb Res 53: 493-501

13. Meade TW (1994) Haemostatic function, arterial disease and the prevention of arterial thrombosis. In: Meade TW (ed) Thrombophilia. Bailliere's Clinical Haematology, vol. 7, n.3. Bailliere Tindall, London, pp 733-755

14. Meade TW, Vickers MV (1985) The effect of physiological levels of fibrinogen on platelet aggregation. Thromb Res 38: 527-534

15. Yamamoto T, Nishiura H, Okamoto M (1995) Participation of transglutaminase in generation of monocyte chemotactic factor. Blood Coagul Fibrinol 6: 341 (Abstract)

16. McDonagh J (1994) Structure and function of factor XIII. In: Colman RW, Hirsh J, Marder VJ, Salzman EW (eds) Hemostasis and thrombosis: basic principles and clinical practice. 3rd edition, JB Lippincott Company, Philadelphia, pp 301-313

17. Sakata Y, Aoki N (1980) Cross-linking of $\alpha-2$ plasmin inhibitor to fibrin by fibrin-stabilizing factor. J Clin Invest 65: 290-297

18. Carmassi F, Chung SI (1983) Regulation of fibrinolysis by factor XIII. In: Davidson JF, Bachman F, Bouvier CA, Kruithof EKO (eds) Progress in fibrinolysis. Churchill Livingstone, Edinburgh, vol. VI, pp 281-285

19. Wiman B (1995) Plasminogen activator inhibitor 1 (PAI-1) in plasma: its role in thrombotic disease. Thromb Haemost 74: 71-76

20. Schneiderman J, Sawdey MS, Keeton MR, Bordin GM, Bernestein EF, Dilley RB, Loskutoff DJ (1992) Increased type 1 plasminogen activator inhibitor gene expression in atherosclerotic human arteries. Proc Natl Acad Scie USA 89: 6998-7002

21. Carmeliet P, Collen D (1995) Gene targeting and gene transfer studies of the plasminogen/plasmin system: implications in thrombosis, hemostasis, neointima formation, and atherosclerosis. FASEB J 9: 934-938

22. Wiman B, Collen D (1977) The specific interaction between plasminogen and fibrin. A physiological role of the lysine binding site in plasminogen. Thromb Res 1: 213-222

23. Lewis MS, Carmassi F, Chung SI (1984) Cooperative association of plasminogen with fibrinogen. Biochemistry 23: 3874-3879

24. Miles LA, Plow EF (1990) Lp(a): an interloper into the fibrinolytic system? Thromb Hemost 63: 331-335

25. Carmassi F, Morale M, Puccetti R, De Negri F, Monzani F, Navalesi R, Mariani G (1992) Coagulation and fibrinolytic system impairment in insulin dependent diabetes mellitus. Thromb Res 67: 643654

26. Juhan-Vague I, Alessi MC, Vague P (1991) Increased plasma plasminogen activator inhibitor-1 levels. A possible link between insulin resistance and atherothrombosis. Diabetologia 34: 457-462

27. Landin K, Tengborn L, Chmielewska J, von Schenck H, Smith U (1991) The acute effect of insulin on tissue plasminogen activator and plasminogen activator inhibitor in man. Thromb Haemost 65: $130-133$

28. Giugliano D, Ceriello A, Paolisso G (1995) Diabetes mellitus, hypertension, and cardiovascular disease: which role for oxidative stress? Metabolism 44: 363-368 